\title{
Multiple Electrodes Active Pulse Echo Method Using a Piezoelectric Film for Crack Identification*
}

\author{
Shiro KUBO**, Takahide SAKAGAMI*** and Toshio SUZUKI** \\ ** Department of Mechanical Engineering, Osaka University \\ 2-1 Yamada-Oka, Suita, Osaka 565-0871 Japan \\ E-mail: kubo@mech.eng.osaka-u.ac.jp \\ *** Department of Mechanical Engineering, Kobe University \\ 1-1, Rokkodai, Nada, Kobe 657-8501 Japan
}

\begin{abstract}
The present authors proposed the smart layer composed of piezoelectric film and flexible printed circuit for the crack identification. The smart layer was used in the passive electric potential CT method, which identifies cracks from the electric potential distribution incurred due to the direct piezoelectric effect on a piezoelectric film pasted on a structure. On the contrary by applying an electric pulse actively on an electrode on the smart layer, a pulse echo can be emitted due to the inverse piezoelectric effect. Reflected waves received at several electrodes can be used for crack identification. In the present study a large number of electrodes on the smart layer were used for emitting the pulse echo and also for receiving the reflected echo. The mono probe method and the dual probe method were proposed. The multiple electrodes active pulse echo method using the smart layer was applied to the identification of through-thickness cracks. It was found that the cracks can be easily detected and a rough estimation of the crack location can be made by receiving flaw echo and bottom echo in the mono probe method. The dual probe method was found useful for detailed identification of the cracks from the time-of-flight of reflected signals for various combinations of the pulsing and receiving electrodes.
\end{abstract}

Key words: Inverse Problem, Crack Identification, Nondestructive Inspection, Experimental Mechanics, Active Pulse Echo Method, Multiple Electrodes, Time-of-Flight Analysis, Piezoelectric Film

\section{Introduction}

Inverse problems and inverse analyses have been receiving increasing attention in various fields of science and engineering. Increasing number of books and review papers on inverse problems are available in the literature [1-8]. Inverse problems can be defined as problems concerning the determination of input or source from output or response. This is contrary to the direct problems, in which output or response are determined using information about input or source. It was found that there are many inverse problems of different types for every research area of science and engineering.

The meaning of inverse problems sometimes depends on research area. The inverse problems can be defined as the problems, which cannot be categorized into direct problems. A rational definition of inverse problems was given by referring to the definition of direct problems $[6,7]$.

*Received 30 Nov., 2011 (No. 11-0738) [DOI: 10.1299/jmmp.6.519]

Copyright () 2012 by JSME 
It was shown that inverse problems can be classified into the following categories of inverse problems.

(A) Estimation of the shape of domain, its boundary or unknown inner boundary (domain/boundary inverse problems).

(B) Inference of the governing equation (governing equation inverse problems).

(C) Estimation of the boundary conditions on the entire or partial boundary and/or estimation of the initial conditions in the domain (boundary value/initial value inverse problems).

(D) Estimation of force or source applying in the domain (force/source inverse problems).

(E) Estimation of material properties defined in the domain and involved in the governing equations (material properties inverse problems).

Any combination of these inverse problems can be another inverse problem.

Due to an increase in the number of aged plants, nondestructive evaluation and online monitoring are gaining importance. The inspection can be regarded as one of the domain/boundary inverse problems, since cracks or defects correspond to unknown inner boundaries to be estimated from certain observations. Ultrasonic inspection, eddy current method, A.C. electric potential method, D.C. potential method, radiation method, dynamic response and strain measurement have been used for the inspection.

The present authors proposed the active electric potential CT (computed tomography) method for the detection and quantitative identification of cracks [9-14]. In this method the electric potential distributions observed on the surface of cracked body under electric current application is used to identify the crack.

The present authors proposed the smart layer composed of piezoelectric film and flexible printed circuit for the crack identification. When the piezoelectric film is glued on cracked body subjected to mechanical load, electric potential distribution is incurred due to the direct piezoelectric effect without applying the electric current on the cracked body. The passive electric potential CT method uses this incurred electric potential [15-20]. Numerical and experimental examinations have shown the effectiveness of the passive electric potential CT method using the piezoelectric film for the crack identification.

On the other hand, when the crack exists far from the top surface, detection became difficult. When electric pulse is input on the piezoelectric film, pulse echo is transmitted due to the inverse piezoelectric effect. The active pulse echo method uses the pulse echo. The simultaneous use of the active pulse echo method and the passive electric potential CT method using the piezoelectric film has shown the effectiveness for the crack identification $[21,22]$.

In this study, the usefulness of the active pulse echo method with multiple electrodes is examined. A large number of electrodes on the smart layer are used for emitting the pulse echo and also for receiving the reflected echo. The multiple electrodes active pulse echo method using the smart layer is applied to the identification of through-thickness cracks. By using the smart layer a pulse echo emitted from many electrodes on the smart layer can be received at many electrodes. The pulse echo for various combinations of the electrodes can be used for the crack identification.

\section{Smart Layer for Active Pulse Echo Method}

The active pulse echo method uses a piezoelectric film pasted on structural material as shown in Fig. 1. When the pulse voltage is applied to the piezoelectric film, the piezoelectric film vibrates due to the inverse piezoelectric effect, and the ultrasonic wave pulse is excited. The incidence pulse echo in the structure is reflected by the boundary like a defect. The reflected ultrasonic pulse is converted into the electric signal on the electrode or on another electrode due to the direct piezoelectric effect. The defect can be identified from 
the reflected signal.

The smart-layer is constructed by accumulating the piezoelectric film, the FPC (Flexible Printed Circuit) that is composed of electrode and wiring pattern as shown in Fig. 1. FPC forms the circuit pattern of conductor foil on the polymer materials such as the polyimide and polyester.

The major advantage of the present method using the smart layer over conventional methods is that the smart-layer can be easily installed since it is thin, light and flexible.

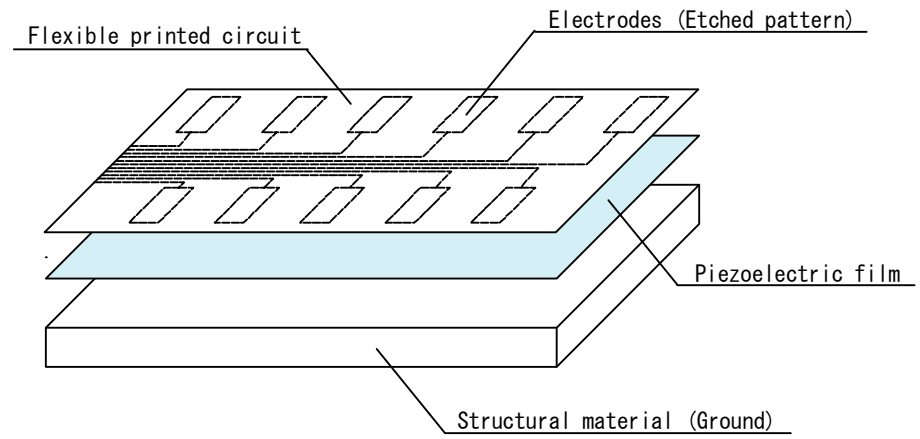

Fig. 1 A schematic illustration of smart-layer pasted on a structural material.

\section{Multiple Electrodes Active Pulse Echo Method}

In the active pulse echo method using multiple electrodes on the smart-layer, the mono probe method and the dual probe method described below are introduced.

\subsection{Mono probe method}

In the mono probe method a pulse echo is emitted from an electrode on the smart layer by applying electric pulse on the electrode, and the echo reflected on the crack surface or on the bottom surface of the structural material is received on the same electrode, as shown in Fig. 2. When there is no crack below the electrode, only a bottom echo is observed at time $t_{\mathrm{nc}}$, which corresponds to the time-of-flight of twice of the distance between the top surface and the bottom surface of the structural material as is shown in Fig. 3. On the contrary, when there is a crack below the electrode, a flaw echo is observed before time $t_{\mathrm{nc}}$. By applying the electric pulse on all the electrodes on the smart layer, the crack can be detected. A rough estimation of the crack area and the depth of the crack can also be made.

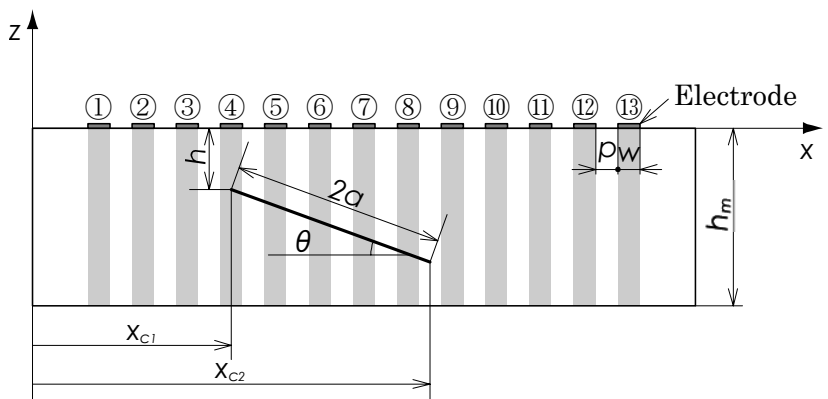

Fig. 2 A schematic illustration of the mono probe method. 


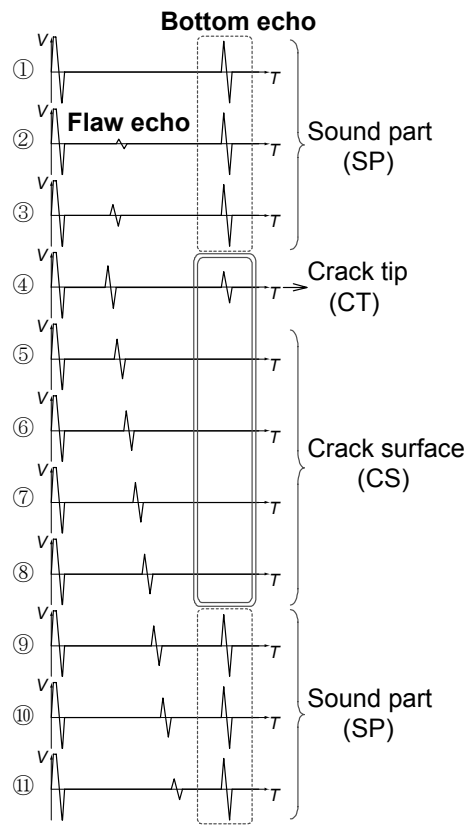

Fig. 3 Reflected signal obtained by the mono probe method.

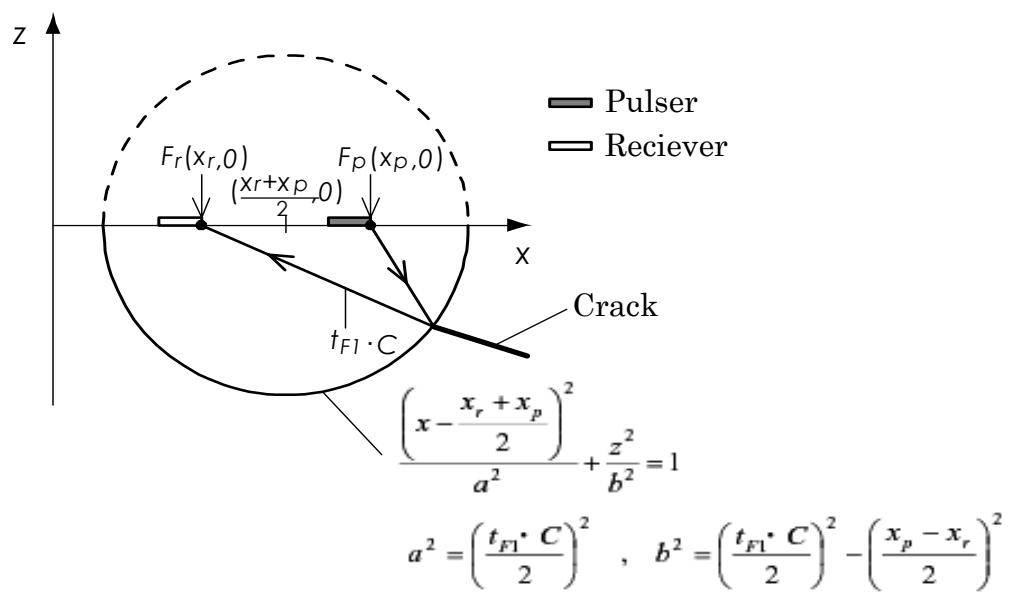

(a) An ellipsoid determined from time-of-flight.

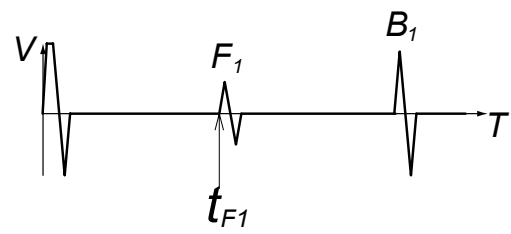

(b) Reflection signal

Fig. 4 Time-of-flight analysis for the dual probe method.

\subsection{Dual probe method}

In the dual probe method a pulse echo is emitted from an electrode on the smart layer by applying electric pulse on the electrode, and the echo reflected on the crack surface or on the bottom surface of the structural material is received on another electrode or on the same 
electrode as is schematically shown in Fig. 4.

Then we can obtain the reflection signal as shown in Fig. 4 (b). From the time-of-flight the summation of the minimum distance from the pulsing electrode to the crack surface and the minimum distance from the crack surface to the receiving electrode is obtained. Then an ellipsoid can be drawn which is tangential to the crack surface. By synthesizing the ellipsoids for various combinations of pulsing electrode and receiving electrode an envelope of the crack can be obtained.

\section{Experiments}

A2024 aluminum specimens with through-thickness crack A and crack B shown in Fig. 5 is used in the experiment. Crack A is parallel to the top surface of the specimen, while crack $\mathrm{B}$ in inclined. The slit was penetrated in the width direction of specimen by electric discharge machining.

Electric pulse was applied to each electrode by the pulse echo method. Considering that the sound wave velocity of the longitudinal wave was large and attenuation was small in the specimen, the applied pulse frequency was set to about $15(\mathrm{MHz})$.

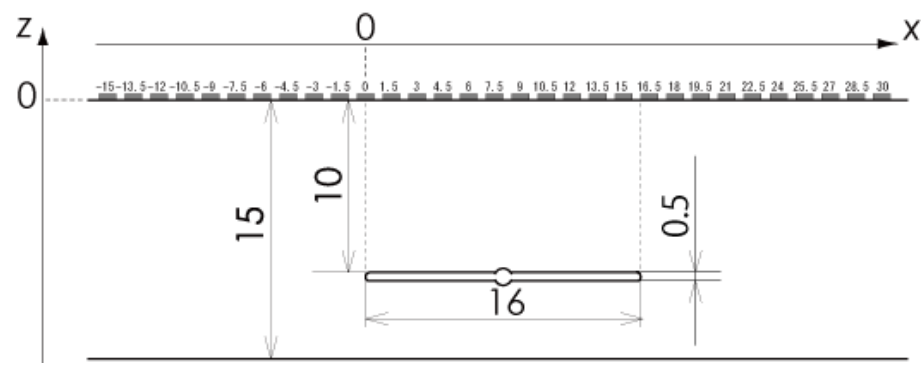

(a) Crack A

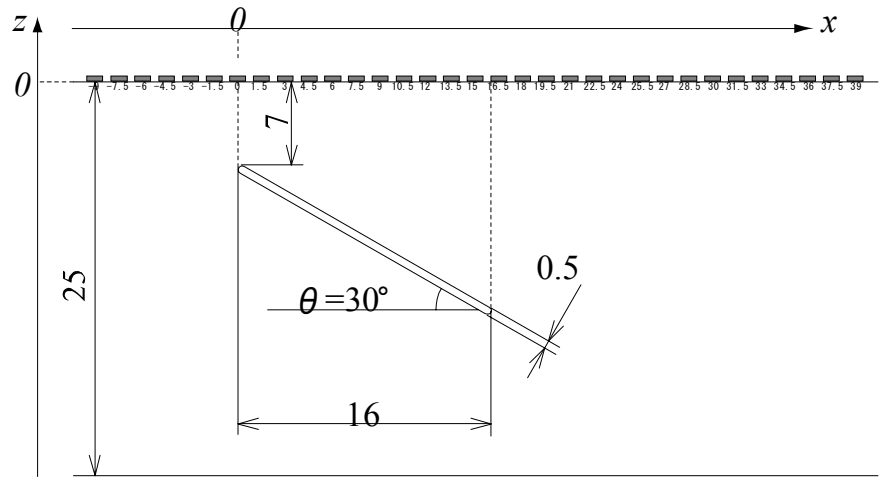

(b) Crack B

Fig. 5 Specimens with a through-thickness crack and smart-layer installed on top surface.

The smart-layer is constructed by accumulating the piezoelectric film and the FPC. The thickness of piezoelectric film is $52(\mu \mathrm{m})$ as shown in Fig. 6. FPC is composed of the electrode formed by etching and the pattern. The FPC pattern has the terminal of lead line. PET (PolyEthylene Terephthalate) film of $50(\mu \mathrm{m})$ in thickness is used as polymer material. 
Aluminum of $10(\mu \mathrm{m})$ in thickness is used as conductor foil. The epoxy adhesive for the strain gauge (EC-30) was used for pasting piezoelectric film with the structural material. The rolling direction of piezoelectric film corresponds to the longitudinal direction of the specimen. Specimen and FPC patterns other than electrode part were electrically insulated by PET film. The size of electrode is $1.0(\mathrm{~mm})$ times $8.0(\mathrm{~mm})$ and the pitch of electrode is $1.5(\mathrm{~mm})$. The two rows of electrode in the longitudinal direction are arranged by shifting the rows by $0.75(\mathrm{~mm})$. The longitudinal direction of the specimen is denoted by $x$. The position of the crack was set to be from $x=0(\mathrm{~mm})$ to16 $(\mathrm{mm})$.

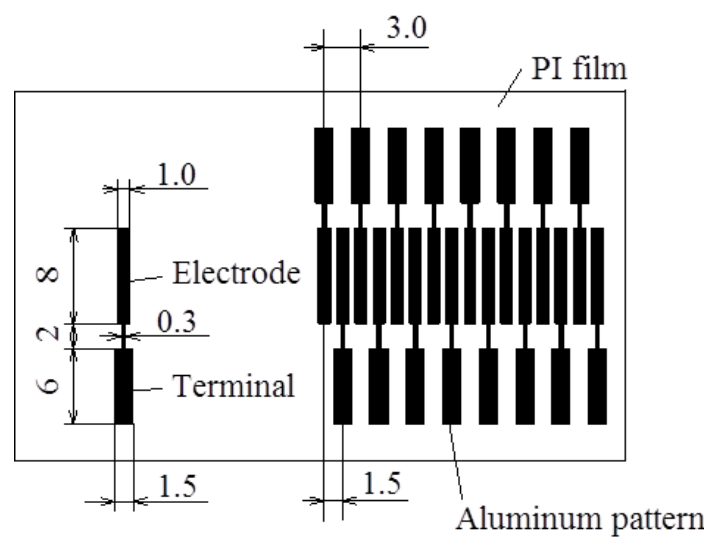

Fig. 6 A pattern of FPC (flexible printed circuit) used in the experiment.

\subsection{Crack A}

\subsubsection{Mono probe method applied to crack $A$}

The mono probe method was applied to the identification of crack A. Figure 7 shows the received echo signal observed by the mono probe method. It is seen that for electrodes locating between $x=0(\mathrm{~mm})$ and $x=15(\mathrm{~mm})$ flaw echo is clearly identified. On the contrary, outside this area significant bottom echo is observed. These indicate that a crack exists in the area between $x=-1.5(\mathrm{~mm})$ and $x=16.5(\mathrm{~mm})$ with the depth of about $10.0(\mathrm{~mm})$. This is in good agreement with the actual location of the crack between $x=0(\mathrm{~mm})$ and $x=16.0$ $(\mathrm{mm})$ with the depth of $10.0(\mathrm{~mm})$. However it is difficult to make detailed estimation of location of crack tips.

\subsubsection{Dual probe method applied to crack $A$}

Dual probe method was applied to the identification of crack A. Figure 8 shows the envelope constructed from the time-of-flight for various combinations of the pulsing electrode and the receiving electrode. It is seen that the envelope agrees well with the actual crack shown by a red line, indicating the effectiveness of the dual probe method.

Table 1 shows a comparison of the estimated location of the crack tips with actual one. It is seen that a very good estimation of the crack location in the longitudinal direction and the depth is made. 


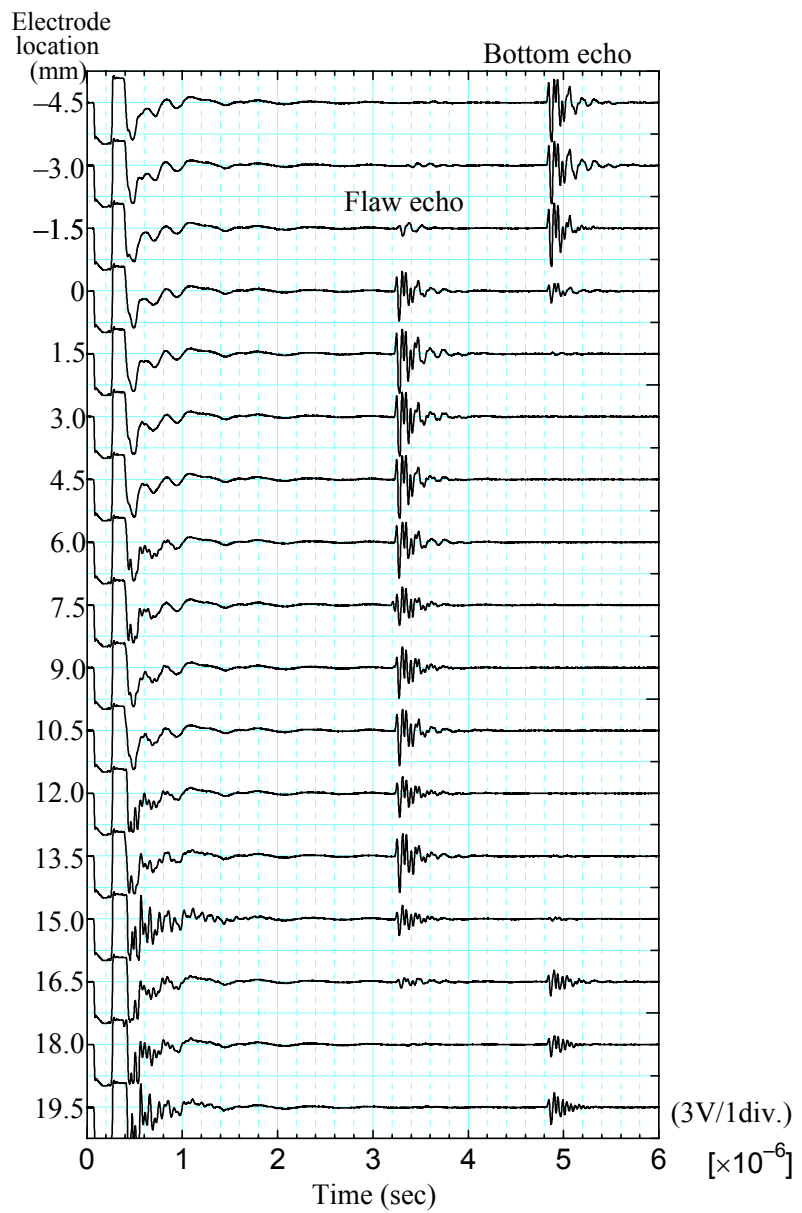

Fig. 7 Reflected signals obtained in the mono probe method for crack A.

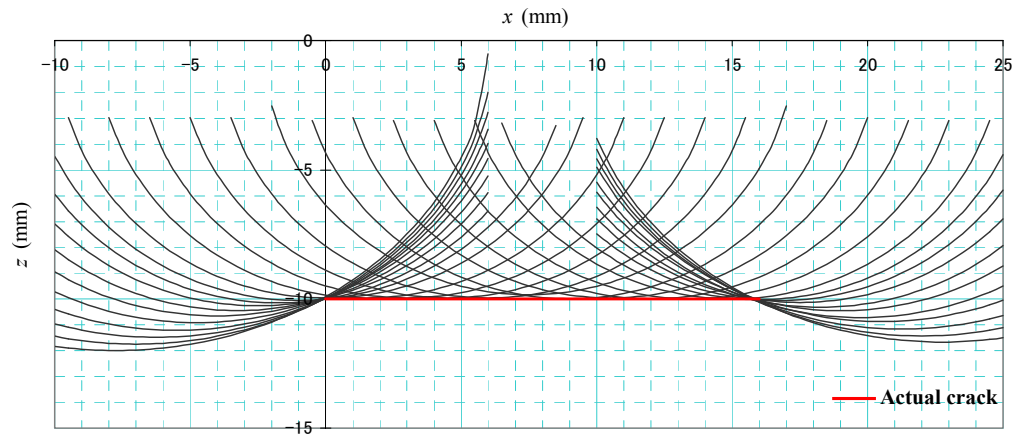

Fig. 8 An envelope constructed by the dual probe method from time-of-flight for various combinations of the pulsing electrode and the receiving electrode for crack $\mathrm{A}$.

Table 1 Estimated value of crack tip locations of crack A.

\begin{tabular}{ccc}
\hline & $\begin{array}{c}\text { Left tip } \\
\left(x_{l}, z_{l}\right)\end{array}$ & $\begin{array}{c}\text { Right tip } \\
\left(x_{r}, z_{r}\right)\end{array}$ \\
\hline \hline Actual & $(0,-10)$ & $(16,-10)$ \\
\hline Estimated & $(0.0,-10.0)$ & $(15.7,-10.1)$ \\
\hline
\end{tabular}




\subsection{Crack B}

\subsubsection{Mono probe method applied to crack B}

The mono probe method was applied to the identification of crack B. Figure 9 shows the received echo signal observed by the mono probe method. It is found that for electrodes locating between $x=0(\mathrm{~mm})$ and $x=15.0(\mathrm{~mm})$ significant bottom echo is not observed and flaw echo is observed. On the contrary, outside this area the bottom echo is observed. These indicate that a crack exists in the area between $x=-1.5(\mathrm{~mm})$ and $x=16.5(\mathrm{~mm})$. This is in good agreement with the actual location of the crack between $x=0(\mathrm{~mm})$ and $x=16.0(\mathrm{~mm})$. However it is difficult to make detailed estimation of location of crack tips.

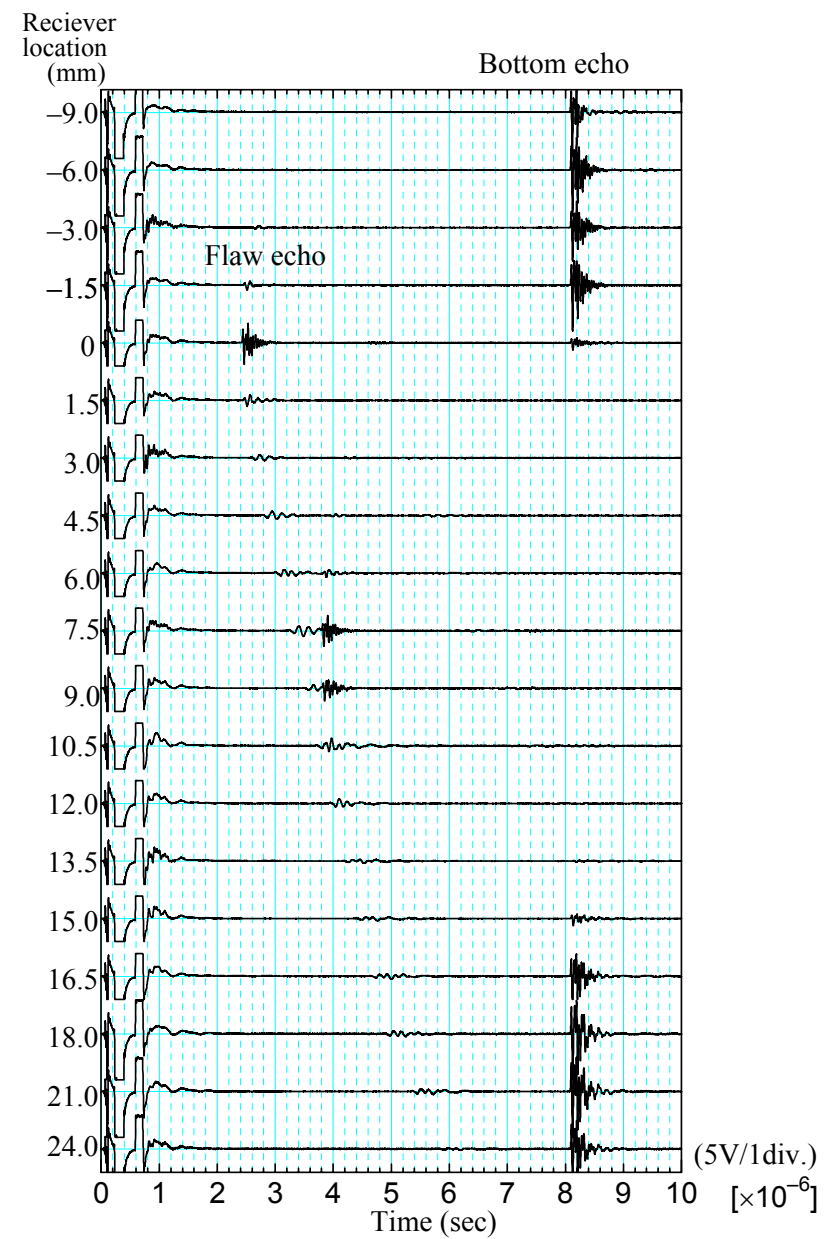

Fig. 9 Reflected signals received at various electrodes obtained in the mono probe method for crack B.

\subsubsection{Dual probe method applied to crack B}

Dual probe method was applied to the identification of crack B. Figure 10 shows an example of the received echo signal observed by the dual probe method for various receiving electrodes. Figure 11 shows the envelope constructed by time-of-flight for various combinations of the pulsing electrode and the receiving electrode. It is seen that the envelope agrees well with the actual crack shown by a red line, indicating the effectiveness 
of the dual probe method.

Table 2 shows a comparison of the estimated location of the crack tips with actual one. It is seen that a very good estimation of the crack location.

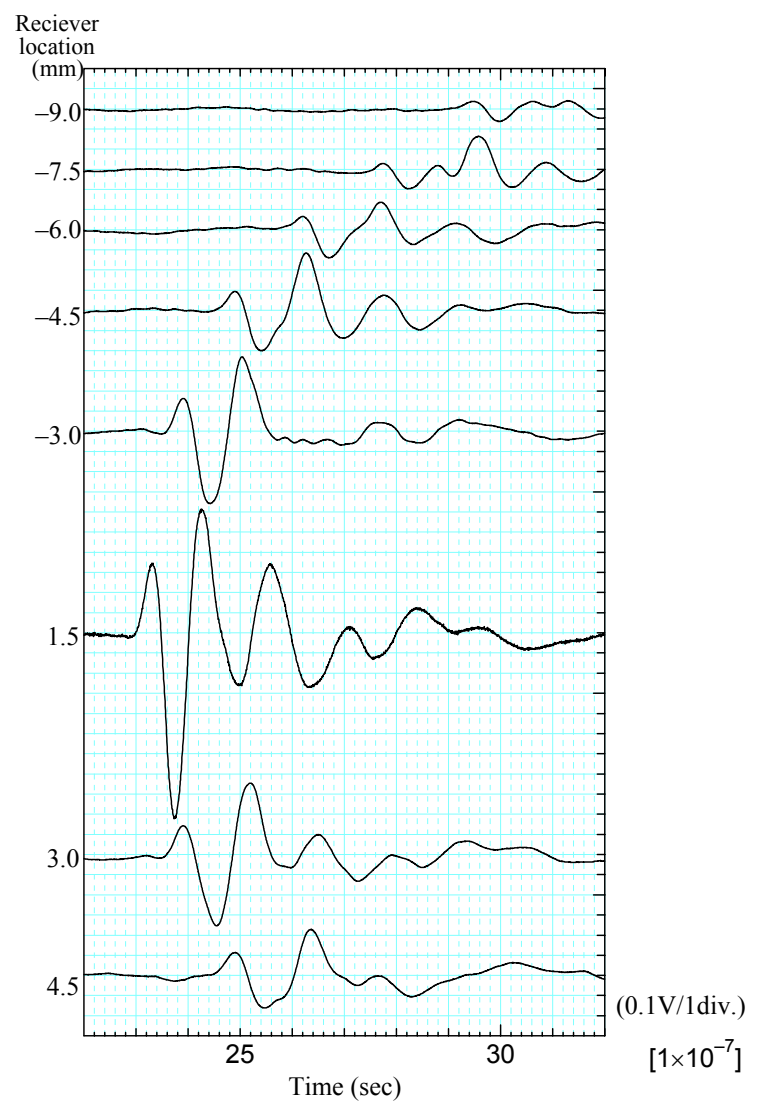

Fig. 10 An example of reflected signals received at various electrodes obtained in the dual probe method for crack B.

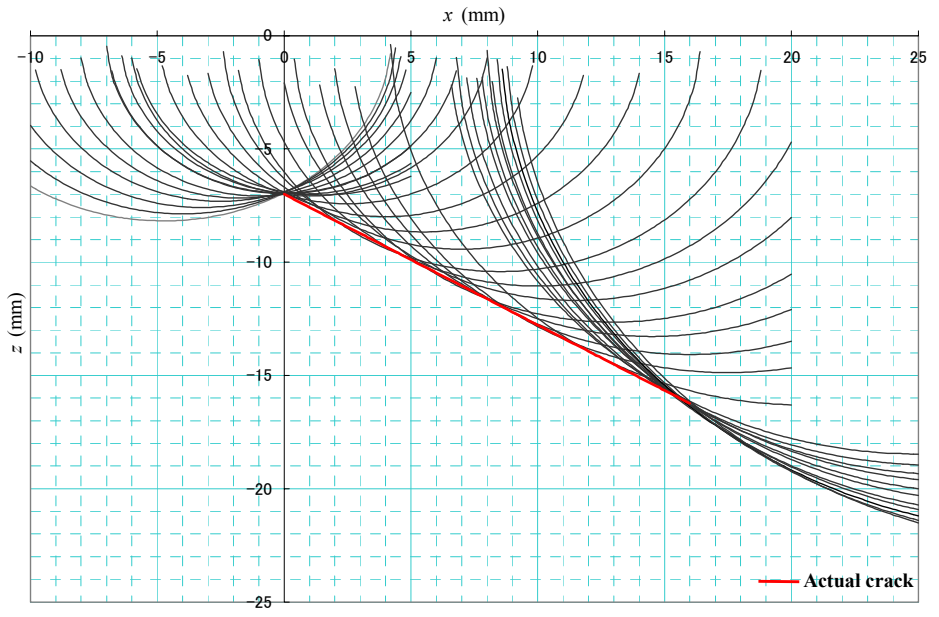

Fig. 11 An envelope constructed by the dual probe method from time-of-flight for various combinations of the pulsing electrode and the receiving electrode for crack B. 
Table 2 Estimated value of crack tip locations for crack B.

\begin{tabular}{ccc}
\hline & $\begin{array}{c}\text { Upper tip } \\
\left(x_{u}, z_{u}\right)\end{array}$ & $\begin{array}{c}\text { Lower tip } \\
\left(x_{l}, z_{l}\right)\end{array}$ \\
\hline \hline Actual & $(0,-7)$ & $(16,-16.2)$ \\
\hline Estimated & $(0.0,-7.0)$ & $(15.8,-16.1)$ \\
\hline
\end{tabular}

\section{Conclusions}

By applying an electric impulse actively on an electrode on the smart layer composed of piezoelectric film and flexible printed circuit, a pulse echo can be emitted. Reflected waves can be received at some electrodes and can be used for crack identification. A large number of electrodes on the smart layer were used for emitting the pulse echo and also for receiving the reflected echo. This multiple electrodes active pulse echo method using the smart layer was applied to the identification of through-thickness cracks.

For the identification, the mono probe method and the dual probe method were proposed. In the mono probe method the pulse echo emitted from an electrode is received by the same electrode. By this method the crack can be detected, and a rough identification of the crack can be made. In the dual probe method a pulse echo emitted from an electrode on the smart layer was received at several electrodes. From the time-of-flight of echo, one can draw an ellipsoid which is tangential to the crack surface. By combining the ellipsoids for various combinations of the pulsing electrode and the receiving electrode, an envelope of the crack can be constituted. The crack can be easily reconstructed from the envelope.

Experimental examination of the active pulse echo method was made for the identification of the through-thickness crack parallel to the top surface and the inclined crack. By applying the mono probe method a rough estimation of the crack was made. By applying the dual probe method an envelope of ellipsoids was determined from the time-of-flight. The envelope agreed well the actual cracks, demonstrating the applicability of the present multiple electrodes active pulse echo method.

\section{Acknowledgments}

This work was partly supported by the Japan Society for the Promotion of Sciences under the Grant-in-Aid for Scientific Research.

\section{References}

[1] Tikhonov, A.N. and Arsenin, V.Y., Solutions of Ill-Posed Problems, John Wiley \& Sons, 1977.

[2] Gladwell, G.M.L., Inverse Problems in Vibration, Martinus Nijhoff Pub., 1986.

[3] Lavrent'ev, M.M., Romonov, V.G. and Shishatskii, S.P., Ill-Posed Problems of Mathematical Physics and Analysis, Amer. Math. Soc., 1986.

[4] Romanov, V.G., Inverse Problems of Mathematical Physics, VNU Sci. Press, 1987.

[5] Groetsch, C.W., inverse Problems in the Mathematical Sciences, Vieweg, 1993.

[6] Kubo, S., and Ohji, K., "Applications to Inverse Problems", in Application of the Boundary Element Method, (in Japanese), Corona Pub., 181-198, 1987.

[7] Kubo, S., "Inverse Problems Related to the Mechanics and Fracture of Solids and Structures”, JSME International Journal, Series I, Japan Soc. Mech. Engrs., 31, 157-166, 1988.

[8] Kubo, S., Inverse Problems, (in Japanese), Baifukan, 1992. 
[9] Kubo, S., Sakagami, T. and Ohji, K., "Electric Potential CT Method for Measuring Two- and Three-Dimensional Cracks", in Current Japanese Materials Research-Vol.8 Fracture Mechanics, Elsevier, Soc. Mat. Sci., Japan, 235-254, 1991.

[10]Kubo, S., Sakagami, T. and Ohji, K., "Electric Potential CT Method Based on BEM Inverse Analyses for Measurement of Three-Dimensional Cracks", Computational Mechanics '86, Springer, 1: V-339-V-344, 1986.

[11]Kubo, S., Sakagami, T. and Ohji, K., "Reconstruction of a Surface Crack by Electric Potential CT Method”, Computational Mechanics '88, Springer, 1: 12.i.1-5, 1988.

[12] Kubo, S., "Requirements for Uniqueness of Crack Identification from Electric Potential Distributions", in Inverse Problems in Engineering Sciences 1990, Springer, 52-58, 1990.

[13] Sakagami, T., Kubo, S., Hashimoto, T., Yamawaki, H. and Ohji, K., "Quantitative Measurement of Two-Dimensional Inclined Cracks by the Electric-Potential CT Method with Multiple Current Applications”, JSME Int. J., Series I, 31, 76-86, 1988.

[14] Kubo, S., Ohji, K. and Aoe, S., "Identification of Plural Cracks by the Electric Potential CT Method Using Fusion-Type Genetic Algorithm", Proc. of Int. Conf. on Advanced Technology in Experimental Mechanics, JSME, 105-110, 1997.

[15]Li, S.Q., Kubo, K., Sakagami, T., and Liu, Z.X, "Theoretical and Numerical Investigations on Crack Identification Using Piezoelectreic Material-Embedded Structures", Materials Sci. Research Int., 6, 41-48, 2000.

[16] Shiozawa, D., Kubo, S. and Sakagami , T., "Passive Electric Potential CT Method Using Piezoelectric Material for Crack Identification", Inverse Problems in Science and Engineering, 12, 71-79, 2004.

[17] Shiozawa, D., Kubo, S. and Sakagami , T., "An Experimental Study on Applicability of Passive Electric Potential CT Method to Crack Identification", JSME International Journal, Series A, 47, 419-425, 2004.

[18] Shiozawa, D., Kubo, S., Sakagami , T. and Takagi, M. "Passive Electric Potential CT Method Using Piezoelectric Material for Identification of Plural Cracks", Computer Modeling in Engineering and Sciences, 11, 27-36, 2006.

[19] Shiozawa, D., Kubo, S. and Sakagami, T., "Experimental Study on Applicability of Passive Electric Potential CT Method for Identification of Three-Dimensional Surface Crack”, JSME International Journal, Series A, 49, 426-435, 2006.

[20] Nakatani, K., Kubo, S., Sakagami, T., Shiozawa, D. and Takagi, M., “An Experimental Study on the Identification of Delamination in a Composite Material by the Passive Electric Potential CT Method", Measurement Science and Technology, 18, No. 1, 2007.1, pp.49-56, 2007.

[21]Kubo, S., Sakagami, T., Suzuki, T., Maeda, T. and Nakatani, K., "Use of the Piezoelectric Film for the Determination of Cracks and Defects -The Passive and Active Electric Potential CT Method", Journal of Physics, Conference Series, 135, 012057, 1-9, 2008.

[22] Kubo, S., Sakagami, T., Ioka,S. and Tsuboi, K., "Effect of Combination of Passive and Active Electric Potential CT Methods Using Piezoelectric Film on Crack Identification", Proc. CST2010: The 10th International Conference on Computational Structures Technology, paper \#65, 1-12, 2010. 